\title{
Eta Carinae's 2014.6 Spectroscopic Event: The Extraordinary He II and N II Features ${ }^{1}$
}

\author{
Kris Davidson ${ }^{2}$, Andrea Mehner ${ }^{3}$,Roberta M.. Humphreys ${ }^{2}$, John C. Martin ${ }^{4}$, and \\ Kazunori Ishibashi ${ }^{5}$
}

\begin{abstract}
Eta Carinae's spectroscopic events (periastron passages) in 2003, 2009, and 2014 differed progressively. He II $\lambda 4687$ and nearby N II multiplet 5 have special significance because they respond to very soft X-rays and the ionizing UV radiation field (EUV). HST/STIS observations in 2014 show dramatic increases in both features compared to the previous 2009.1 event. These results appear very consistent with a progressive decline in the primary wind density, proposed years ago on other grounds. If material falls onto the companion star near periastron, the accretion rate may now have become too low to suppress the EUV.
\end{abstract}

Subject headings: stars: individual ( $\eta$ Carinae) - stars: winds, outflows - stars: massive - stars: variables: general - circumstellar matter - X-rays: stars

\section{He II $\lambda 4687$ emission in $\eta$ Car's spectroscopic events}

The appearance of $\eta$ Car has evolved rapidly in the past 15 years (Martin et al. 2006a, 2010; Mehner et al. 2010b, 2012). The primary wind may be returning to its pre-eruption state (Davidson 2012; Humphreys et al. 2008), but in any case the spectrum and brightness have changed much faster than before. Extraordinary clues are provided by "spectroscopic events" that occur near each periastron passage of the companion star in its 5.5-year

\footnotetext{
${ }^{1}$ Based on observations made with the NASA/ESA Hubble Space Telescope, which is opera ted by the Association of Universities for Research in Astronomy, Inc., under NASA contract NAS 5-26555.

${ }^{2}$ Minnesota Institute for Astrophysics, 116 Church St SE, University of Minnesota, Minneapolis, MN 55455

${ }^{3}$ ESO, Alonso de Cordova 3107, Vitacura, Santiago de Chile, Chile

${ }^{4}$ Barber Observatory, University of Illinois, Springfield, IL, 62703

${ }^{5}$ Division of Elementary Particle Physics and Astrophysics, Graduate School of Science, Nagoya University, Nagoya, 464-8602, Japan
} 
orbit - see many refs. in Corcoran \& Ishibashi (2012), Humphreys \& Martin (2012), and Davidson (2012). The events observed in 1998, 2003, and 2009 did not match each other (Davidson et al. 2005; Kashi \& Soker 2009b; Mehner et al. 2011b). Here we report major differences between the 2014.6 event and its predecessors, observed with the Space Telescope Imaging Spectrograph (HST/STIS).

The 2003.5 and 2009.1 spectroscopic events seemed alike in their early stages, but their X-rays and exotic He II emission differed after periastron. Broad He II $\lambda 4687$ emission occurred during the first half of each event (Steiner \& Damineli 2004; Martin et al. 2006b; Mehner et al. 2011b; Teodoro et al. 2012), even though neither star can ionize $\mathrm{He}^{+}$enough to produce this recombination line. Martin et al. noticed its anti-correlation with the $2-10 \mathrm{keV}$ X-rays, and explored relevant physics. They concluded that a flood of 50-700 eV photons arose as the colliding-wind shocks became unstable near periastron, indirectly exciting He II $\lambda 4687$. Those soft X-rays carry far more energy flux than the $2-10 \mathrm{keV}$ photons, but cannot be observed except via He II $\lambda 4687$.

In 2009, unlike 2003, definite $\lambda 4687$ emission briefly reappeared after periastron. (Concerning the 2003 record, see $\S 5$ below.) The hard X-rays reappeared immediately thereafter, much earlier than in 1998 and 2003. Most likely, $\lambda 4687$ emission signaled the re-formation of large-scale shock structure which produces hard X-rays. These and other data appear consis-

tent with lower gas densities in 2009 compared to 2003 (Kashi \& Soker 2009b; Mehner et al. 2010b, 2011b; Corcoran et al. 2010).

Thus, a qualitative extrapolation of the 1998-2003-2009 data suggested that $\eta$ Car's 2014.6 event might have a brighter He II $\lambda 4687$ "second flash." Here we report HST/STIS observations which confirm that suspicion. Equally significant, they also reveal unexpected strength in a nearby UV-excited N II multiplet that was only barely visible in 2003 and 2009.

\section{Observations and Data Reduction}

HST /STIS plays a unique role in our knowledge of $\eta$ Car. Unlike ground-based spectrographs, STIS can observe the central object without serious contamination by ejecta located 150-500 mas from the star (Hamann 2012; Remmen et al. 2012; Martin et al. 2006a). It also provides unrivaled data homogeneity over the time period 1998-2014, immune to atmospheric effects - though it was not operational during the 2009.1 event. Moreover, STIS allows UV coverage. For all these reasons, HST data provide necessary anchoring points for ground-based observations (see $§ 5$ below).

The STIS/CCD observations reported here cover wavelength range 4562-4841 $\AA$ with 
a 100 mas slit width and an integration time of $25.5 \mathrm{~s}$ on each occasion. The observing dates in 2014 are listed in Table 1. The instrument setup was like that described in our earlier papers, especially Martin et al. (2006b). Details can be found in the STScI and $\eta$ Car Treasury Program archives, where the data will become publicly available in 2015.1 Additional wavelength ranges will be reported in later papers, but He II $\lambda 4687$ and the special N II emission are most critical.

We used the data reduction methods employed by Martin et al. (2006b) and Mehner et al. (2010b), developed for the HST $\eta$ Car Treasury Project in 2002-2004 - providing better spatial resolution than the standard STScI pipeline (Davidson 2006). They allowed us to extract reliable spectra with spatial widths of 150 mas and slightly unsharp edges to minimize pixelization effects. At $\eta$ Car's distance, 150 mas corresponds to a projected size of about 340 AU, larger than the companion star's orbit but small enough to exclude the bright ejecta knots which contaminate all ground-based spectra of this object. Spectral resolution was roughly $40 \mathrm{~km} \mathrm{~s}^{-1}$, much narrower than the features discussed below. Since each relevant CCD pixel had many thousands of counts, and each spectral feature included many pixels, statistical errors are negligible compared to systematic errors. With HST's excellent spatial resolution, systematic effects result mainly from the complexity of $\eta$ Car's spectrum, unrelated to the instrument.

Regarding "phase" in the 5.5-year orbital cycle, we use the definition adopted long ago for the $\eta$ Car Treasury Project: period $=2023.0$ days exactly, and phase $=0$ at MJD 56883.0 (2014 August 14), MJD 54860.0 (2009 January 29), etc. Advantages of this system are outlined by Mehner et al. (2011b). We express $t$, time from the nearest zero phase, in days. The time of periastron is unknown but most likely occurs near $t \sim-10 \mathrm{~d}$.

Quoted wavelengths are in vacuum, and Doppler velocities are heliocentric.

\section{He II $\lambda 4687$ in 2014}

These observations were intended to answer two obvious questions: (1) Was there a substantial difference between the 2014.6 and 2009.1 events concerning He II $\lambda 4687$ ? (2) If so, was the post-periastron emission stronger in 2014, extrapolating the 2003-2009 difference? Both answers prove to be "yes."

For comparison purposes, Figure 1 shows typical STIS spectra during and after the 2003.5 event, and in September 2013. In addition, the bottom panel shows a Gemini/GMOS

\footnotetext{
1 http://archive.stsci.edu/hst/ and http://etacar.umn.edu/.
} 
observation in 2009 (Mehner et al. 2011b). It has better spatial resolution than most other ground-based data, but nevertheless shows major extraneous features due to outlying ejecta. (Compare it to the top panel in Fig. 1 and the second panel in Fig. 2, obtained at similar orbital phase.)

The critical range 4675-4694 $\AA$ is marked by vertical lines in Fig. 1. STIS sampled this wavelength region on 15 occasions outside the spectroscopic events from 1998 to 2012, and none of them showed any sign of the $\lambda 4687$ feature on the star - see, e.g., Figs. $3-6$ in Martin et al. (2006b). They resembled 2003.88 in Figure 1 and 2014.85 in Figure 2, except that most other emission lines progressively weakened from 2000 to 2012 (Mehner et al. 2010b, 2012). The 2013.70 spectrum in Figure 1 shows two noteworthy departures from the earlier record: a N II multiplet around $4620 \AA$ had become conspicuous in absorption ( $\S 4$ below), and a possible weak emission bump can be seen near $4687 \AA$.

Figure 2 shows our recent STIS spectra. Elevated fluxes near $4687 \AA$ - the shaded areas in the figure - occur only during spectroscopic events.

Results for the $\lambda 4687$ emission strength appear in Figure 3. This is a contentious topic, and the chief issue is whether the emission differed substantially between 2014.6 and 2009.1. Therefore we employ two largely independent measures of the emission strength and they both support the same conclusion. EW1 samples only the the "obvious bump" in the spectrum with a peak near $4687 \AA$ (e.g., top panel in Figs. 1 and 2); this is easy to measure but it omits much of the flux. EW2 includes a larger fraction of the emission but requires an accurate measure of the true continuum.

For EW1 we simply estimate a linear pseudo-continuum based on data within the narrow range 4670-4700 $\AA$, and calculate net equivalent width in the usual way. A dashed line in the top panel of Figure 1 shows an example. EW1 amounts to a lower limit, because its pseudocontinuum level often lies well above the true continuum. EW1 in the 2009.1 and 2014.6 events is shown in Figure 3a, where small data points represent Gemini/GMOS observations in 2009 (Mehner et al. 2011b), while heavier black dots are the STIS results for 2014. Limit marks on each STIS data point are not standard error bars; instead they indicate a range of $\pm 1.5 \%$ in the local pseudo-continuum. Levels outside this range do not appear credible when plotted with the data. Likely errors are difficult to quantify because they are mainly systematic, but the limit marks in Fig. 3 might be regarded as informal equivalents to normal $1.5 \sigma$ or $2 \sigma$ errors.

During the first half of each spectroscopic event, EW1 was noticeably larger in 2014 compared to 2009 (Fig. 3a). But the second half of the event, particularly $t \approx+17 \mathrm{~d}$, showed a more dramatic difference: the second flash in 2014 was roughly twice as strong as 
its predecessor in 2009.

Figures 2 and 3b show that EW1 is less than half of the story, because broader emission fills the region around $4687 \AA$ during an event. Martin et al. (2006b) showed that flux levels near 4605 and $4745 \AA$ measured $\eta$ Car's continuum sufficiently well in the 1998-2004 STIS data. When He II $\lambda 4687$ was not present (i.e., at any time outside an event), linear continua $f_{\lambda}^{c}$ fit the 4605, 4685, and $4745 \AA$ levels very well; e.g., 2003.88 in Fig. 1. The apparent slope was small, with $f_{\lambda}^{c}(4685 \AA) \approx 0.99 f_{\lambda}^{c}(4745 \AA) 2$ Estimating the continuum in this way, and integrating the emission between 4675 and $4694 \AA$, we find a second equivalent width EW2 which is often much larger than EW1. Martin et al. (2006b), Mehner et al. (2011b), and Teodoro et al. (2012) used this procedure. Emission outside the 4675-4694 $\AA$ interval is blended with strong unrelated spectral features.

In 2014, a set of unusual N II lines disqualified the $4605 \AA$ window for continuum sampling (Fig. 2 and $\S 4$ ). We must therefore base the underlying continuum on just the 4745 $\AA$ window plus earlier experience and known features. Since $4687 \AA$ and $4745 \AA$ differ by only $1.2 \%$ in wavelength, no likely process would alter the continuum ratio $f_{\lambda}^{c}(4687 \AA) / f_{\lambda}^{c}(4745 \AA)$ by more than a fraction of a percent. (4687 $\AA$ is on the insensitive Rayleigh-Jeans side of $\eta$ Car's SED.)

We estimate EW2 values in the following way, favoring slightly higher continuum to avoid exaggerating the 2014.6 event. First, we assume that $f_{\lambda}^{c}(4745 \AA)$ is $1 \%$ higher than the average of $f_{\lambda}$ across several $\AA$ near $4745 \AA$. This correction is motivated by two weak absorption lines near 4750 and $4763 \AA$ (see below). Then we adopt $f_{\lambda}^{c}(4687 \AA)=f_{\lambda}^{c}(4745 \AA)$, integrate $f_{\lambda}-f_{\lambda}^{c}$ from 4675 to $4694 \AA$, and calculate equivalent width EW2 accordingly. Very likely the two weak absorption lines depress $f_{\lambda}^{c}(4745 \AA)$ by less than $1 \%$, and earlier experience suggests that $f_{\lambda}^{c}(4687 \AA)$ is about $1 \%$ less than $f_{\lambda}^{c}(4745 \AA)$. Consequently our EW2 values may be roughly $0.3 \AA$ too low, i.e., we err on the side of skepticism.

In terms of EW2, Figure 3b shows that the second $\lambda 4687$ flash in 2014 was more than twice as strong as in 2009. The small horizontal bar above each data point shows the value if we decrease $f_{\lambda}^{c}(4687 \AA)$ by $1 \%$, arguably more likely as noted above.

Various objections to our measurements can be proposed, but they are weak when examined. The temporal sampling is adequate to establish the brightening, since all data in 2009 showed a continuous rise and decline through about 45 days. Our 2014 observations sampled the rise, the near-maximum, and the decline, and all three were much brighter

\footnotetext{
2 The precise apparent slope is slightly influenced by focus effects (Davidson 2006); but what matters here is long-term consistency, which has been excellent with STIS.
} 
than corresponding phases in 2009. (If the true maximum was substantially higher, then the 2009-2014 increase is even larger than we report.) We cannot prove that extra emission in 4675-4694 $\AA$ (shaded in Fig. 2) was entirely due to He II; but something changed in EW2 between 2009 and 2014, and it correlated with EW1 which certainly represents He II emission. Errors in the continuum slope cannot be large enough to affect the issue, for physical reasons mentioned above. No evident process can enhance the continuum in a wavelength range less than $100 \AA$ wide. The narrow interval near $4745 \AA$ is indeed the best continuum sample, because emission and absorption features are known to affect all other wavelengths between 4550 and $4800 \AA$ during the spectroscopic event. A Thomson-scattered wing of He I $\lambda 4714$ cannot account for much of EW2, because that would entail a detectable slope in $f_{\lambda}$ around $4687 \AA$; and, also, the corresponding long-wavelength wing is not strong enough. Altogether, EW2 is well justified and EW1 behaved similarly. Conceivably the values for 2009 were severely underestimated because of ground-based defects mentioned above, but that would not explain why the second flash around $t \sim+20 \mathrm{~d}$ changed far more than the first flash around $t \sim-30 \mathrm{~d}$.

In summary, the combination of EW1 and EW2 in Figure 3 establishes a conspicuous difference between the 2014.6 and 2009.1 events. Its physical significance will be noted in $\S 5$ and in later papers.

\section{Special N II features and the EUV}

N II multiplet 5 at 4603-4644 $\AA$, barely noticeable in 2009, became quite conspicuous in 2014; compare especially the 2014.57 panel in Figure 2 to any spectrum in Figure 1 . This development indicates a strengthened EUV radiation field. The multiplet's lower level has

energy $18.5 \mathrm{eV}$, far too high for normal thermal excitation. As explained by Mehner et al. (2011a), it is populated in $\eta$ Car's primary wind by photons from the hot companion star. Consequently multiplet 5 can scatter ambient radiation, thus producing absorption and/or pseudo-emission lines, depending on viewing direction.

In late 2013 this set of features appeared mainly in absorption (2013.70 in Fig. 1), but during the 2014.6 event they became mainly emission (Fig. 2). Mehner et al. sketched the geometrical circumstances in their Figure 5. In late 2013 the secondary star passed between us and the primary, except for the orbit inclination. Thus it excited N II along our line of sight to the primary wind, causing absorption in multiplet 5. In mid-2014 the secondary star moved to the far side of the primary, unfavorable for absorption but allowing apparent emission which was really light scattered in gas around the sides of the configuration. By November 2014 the radiative excitation weakened because the size scale had increased. 
Why were these features strong in 2014 but not in 1998, 2003, and 2009? N II multiplet 5 requires photons with $h \nu \gtrsim 14.5 \mathrm{eV}$ to create $\mathrm{N}^{+}$, and $h \nu \approx 18.5 \mathrm{eV}$ to excite it. Normally these come from the hot secondary star. During the earlier spectroscopic events, its UV was effectively suppressed (Zanella et al. 1984, and other refs. in Davidson 2012). Evidently the 2014.6 event was characterized by a stronger EUV radiation field, presumably from the secondary star.

The N II velocities vary in much the same way as some other features (Mehner et al. $2011 \mathrm{a}$, b). We do not discuss their kinematics here, because their strength has a more immediate significance as outlined above.

\section{Discussion}

A wealth of evidence affirms the rapid and progressive changes in $\eta$ Car since 1998; see Mehner et al. (2012) and many refs. therein. But this development has occasionally been disputed (e.g. Damineli et al. 2010; Teodoro et al. 2012; Madura et al. 2013). For He II $\lambda 4687$, Teodoro et al. (2014) report "no significant changes between 2009.0 and 2014.6." Figure 3 contradicts that assessment, and the two events differed in other respects concerning the UV radiation field. The most natural explanation continues to be a progressive decline of the primary wind density, see many references in Davidson (2012) and Mehner et al. (2012). Since the photosphere is located in the wind, decreasing densities favor smaller photospheric radii, higher radiation temperatures, and higher ionization states.

Whatever caused the "extra emission" around $4687 \AA$ (shaded areas in Fig. 2), it manifestly correlates in time and wavelength with the undoubted He II $\lambda 4687$ feature measured by EW1. In the absence of any other credible candidate, broadened He II emission is the simplest interpretation and its physics appears reasonable (Martin et al. 2006b). Column densities near periastron (Corcoran \& Ishibashi 2012) are large enough to produce Thomson scattering, which broadens some of the $\lambda 4687$ emission but also reflects much of it away from us. The $\lambda 4687$ minimum around $t \sim-10 \mathrm{~d}$ may conceivably have been a quasi-eclipse by gas near the primary star, and the "second flash" may have been stronger in 2014 merely because intervening densities were lower than in 2009. Quantitative models will require elaborate simulations of colliding-wind shock instability, breakup, and reformation, and there are more adjustable parameters than truly independent observables. The main point at present is that $\eta$ Car's successive spectroscopic events do indeed differ in a progressive way.

Subtle effects in ground-based data have occasionally led to misunderstandings. For example, Teodoro et al. (2012) stated that weak He II $\lambda 4687$ persisted between events, with 
a strength that would have been obvious in the STIS data. In fact this was presumably emission from outlying ejecta at $r \sim 0.3$ to 1 arcsec, as explained in $\S 4.2$ of Mehner et al. $(2011 \mathrm{~b})$ and $\S 6.3$ of Martin et al. (2006b). Teodoro et al. also stated that a second $\lambda 4687$ flash occurred in 2003, based on two or three instances of EW $\sim 0.6 \AA$ from a relatively modest instrument. Between those observations, however, STIS showed EW2 $<0.2 \AA$ at $t$ $=+15 \mathrm{~d}$. Teodoro et al. speculated that this was a "bad datum," which is highly unlikely in view of the proven nature of the instrument and the multiple consistency of Fig. 5 in Martin et al. (2006b). Since STIS was the more capable instrument, and hard X-rays did not reappear soon after that time, most likely there was no strong $\lambda 4687$ flash near $t \sim+15$ d in 2003.

We mention these examples because they illustrate why excellent data with high spatial resolution are essential for this problem. The bottom panel in our Figure 1, and also Figure 2 in Teodoro et al. (2012), show that even the best ground-based spectra of $\eta$ Car have obvious contamination by outlying narrow-line ejecta. (Compare them to the STIS spectra.) Data obtained with wider slits or inferior atmospheric conditions may be appreciably worse; and the relative brightness of the ejecta is changing with time (Mehner et al. 2012). The extraneous features cast doubt on continuum estimates and other sensitive measures that rely only on ground-based data. STIS, by contrast, has far better spatial resolution, excellent $\mathrm{S} / \mathrm{N}$, and good long-term stability; see especially $§ 4.2$ in Mehner et al. (2011b). Moreover, its data are publicly available.

N II multiplet 5 samples different physical parameters than either the helium lines or the low-excitation features. It depends mainly on EUV photons with energies between 14 and $20 \mathrm{eV}$, supplied by the hot companion star (Mehner et al. 2011a). At most times the secondary star has $T_{\text {eff }} \sim 40000 \mathrm{~K}$, producing copious radiation at $14-20 \mathrm{eV}$ (Mehner et al. 2010a). The N II features arise in relatively normal parts of the primary wind, not the shocked regions. Their prominence in 2014 implies a substantial EUV radiation field.

Traditionally, $\eta$ Car's spectroscopic events were defined by a temporary lack of ionizing UV (Zanella et al. 1984). Either (1) the hot secondary star moved inside the diffuse primary photosphere, and/or (2) it accreted primary-wind material near periastron, thereby reducing its $T_{\text {eff }}$ (Soker 2003, 2005, 2007; Kashi \& Soker 2009a,b). Both of these possibilities require ambient densities above some critical level. Therefore, the unpredicted strength of N II multiplet 5 in 2014 strongly suggests that gas densities were lower than they were in previous events, consistent with a progressively decreasing primary wind (Mehner et al. 2010b, 2011b; Davidson 2012 and refs. therein). In any case it signals another physical difference between the 2014.6 event and its predecessors.

Additional HST/STIS results on $\eta$ Car's 2014.6 event will be reported in subsequent 
papers.

Acknowledgements: As always, we are grateful to Beth Periello and other STScI staff members for help in scheduling the intricate STIS observations. These data swere obtained in HST program GO 13377, whose P.I. is A. Mehner. We also thank Gary Ferland for good advice concerning line identifications and related tasks.

\section{REFERENCES}

Corcoran, M. F., Hamaguchi, K., Pittard, J. M., Russell, C. M. P., Owocki, S. P., Parkin, E. R., \& Okazaki, A. 2010, ApJ, 725.1528

Corcoran, M.F., \& Ishibashi, K. 2012, in Eta Carinae and the Supernova Impostors, Astrophys. \& Sp. Sci. Library 384 (ed. K. Davidson \& R.M. Humphreys, Springer Media, New York), 195

Davidson, K., Martin, J.C., Humphreys, R.M., et al. 2005, AJ, 129, 900

Davidson, K. 2006, in The 2005 HST Calibration Workshop: Hubble After the Transition to Two-Gyro Mode, ed. A. M. Koekemoer, P. Goudfrooij, \& L. L. Dressel, 247

Davidson, K. 2012, in Eta Carinae and the Supernova Impostors, Astrophys. \& Sp. Sci. Library 384 (ed. K. Davidson \& R.M. Humphreys, Springer Media, New York), 43

Damineli, A., Teodoro, M., \& Groh, J.H. 2010, in Active OB Stars, IAU Symp. 272 (ed. C. Neiner, G. Wade, G. Meynet \& G. Peters), 119

Hamann, F. 2012, in Eta Carinae and the Supernova Impostors, Astrophys. \& Sp. Sci. Library 384 (ed. K. Davidson \& R.M. Humphreys, Springer Media, New York), 95

Humphreys, R. M., Davidson, K., \& Koppelman, M. 2008, AJ, 135, 1249

Humphreys, R.M., \& Martin, J.C. 2012, in Eta Carinae and the Supernova Impostors, Astrophys. \& Sp. Sci. Library 384 (ed. K. Davidson \& R.M. Humphreys, Springer Media, New York), 1

Kashi, A. \& Soker, N. 2009, New Astronomy, 14, 11

Kashi, A. \& Soker, N. 2009, ApJ, 701, L59

Madura, T.I., Gull, T.R., Okazaki, A.T., et al. 2013, MNRAS, 436, 3820

Martin, J. C., Davidson, K., \& Koppelman, M. D. 2006a, AJ, 132, 2717 
Martin, J. C., Davidson, K., Humphreys, R. M., Hillier, D. J.,\& Ishibashi, K. 2006b, ApJ, 640,474

Martin, J. C., Davidson, K., Humphreys, R. M. \& Mehner, A. 2010, AJ, 139, 2056

Mehner, A., Davidson, K., Ferland, G.J., \& Humphreys, R.M. 2010, ApJ, 710, 729

Mehner, A., Davidson, K., Humphreys, R. M., Martin, J. C., Ishibashi, K., Ferland, G. J. \& Walborn, N. R. 2010, ApJ, 717, L22

Mehner, A., Davidson, K., \& Ferland, G. J. 2010, ApJ, 737:70

Mehner, A., Davidson, K., Martin, J. C., Humphreys, R. M., Ishibashi, K., \& Ferland, G. J. 2011, ApJ, 740:80

Mehner, A., Davidson, K., Humphreys, R. M., Ishibashi, K., Martin, J. C., Ruiz, M. T., \& Walter, F. M. 2012, ApJ, 751:73

Remmen, G., Davidson, K., \& Mehner, A. 2012, ApJ, 773:27

Soker, N. 2003, ApJ, 597, 513

Soker, N. 2005, ApJ, 635, 540

Soker, N. 2007, ApJ, 661. 482

Steiner, J.E. \& Damineli, A. 2004, ApJ, 612. L133

Teodoro, M., Damineli, A., Arias, J.I., et al. 2012, ApJ, 746:73

Teodoro, M., Heathcote, B., Richardson, N., et al 2014, The Astronomers' Telegram, ATEL 6464

Zanella, R., Wolf, B., \& Stahl, O. 1984, A\&A, 137, 79 


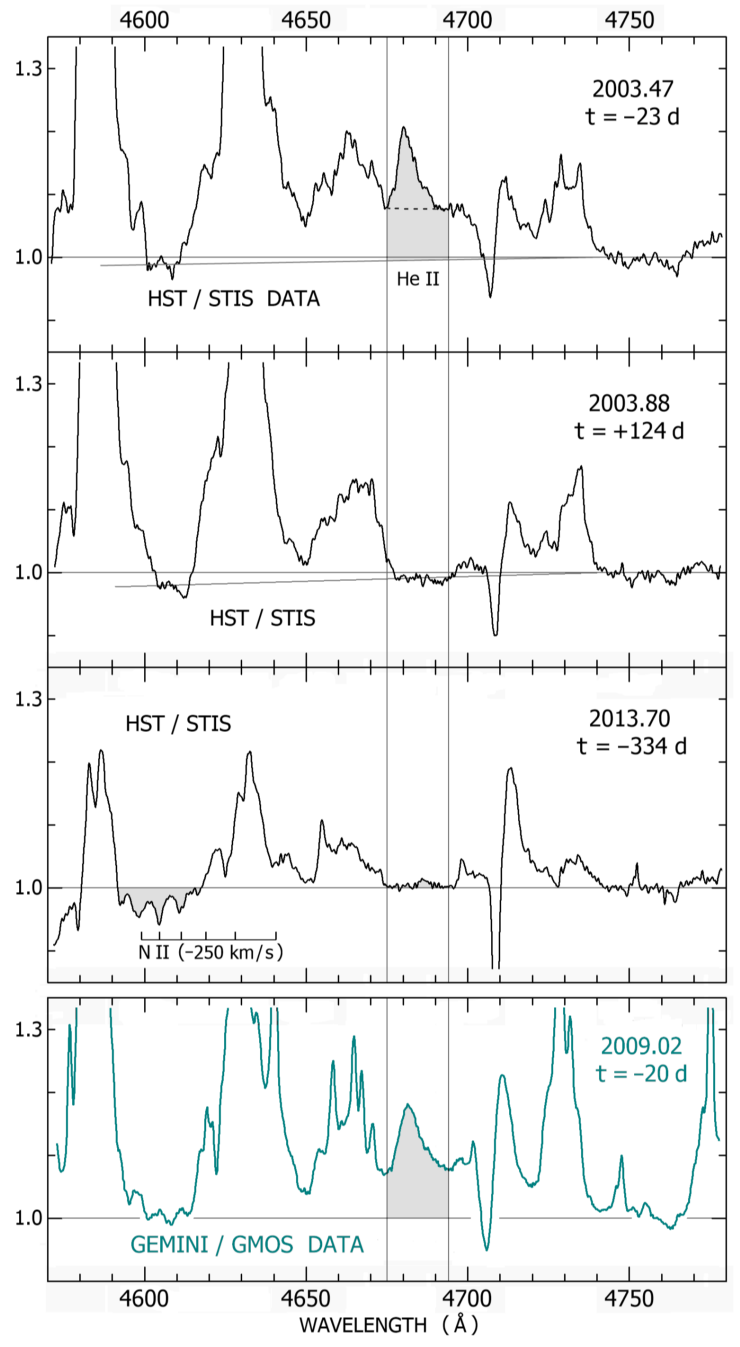

Fig. 1. - Representative STIS data in 2003 and 2013, and ground-based data in 2009. Each tracing shows $f_{\lambda}$ relative to the adopted value near $4745 \AA$. Baselines with small slopes in the 2003.47 and 2003.88 panels indicate continuum fits. A short dashed line in the 2003.47 panel shows the base for a typical EW1 estimate, see text. The bottom panel shows Gemini/GMOS data in the first half of the 2009 event; note the weakness of N II features around $4610 \AA$ compared to Fig. 2. Like all other ground-based spectra of $\eta$ Car, the Gemini data include many features emitted by outlying ejecta. 


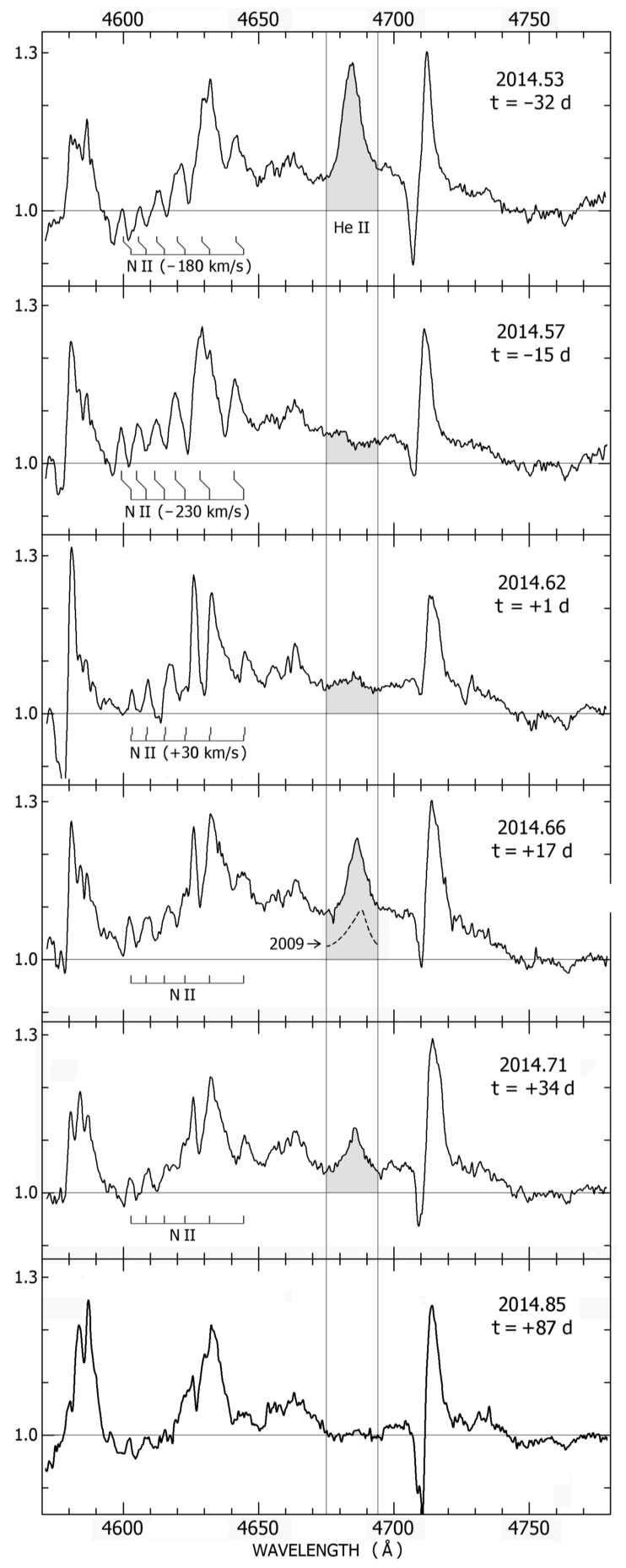

Fig. 2.- STIS data in $2014, f_{\lambda}$ relative to the adopted continuum value near $4745 \AA$. 


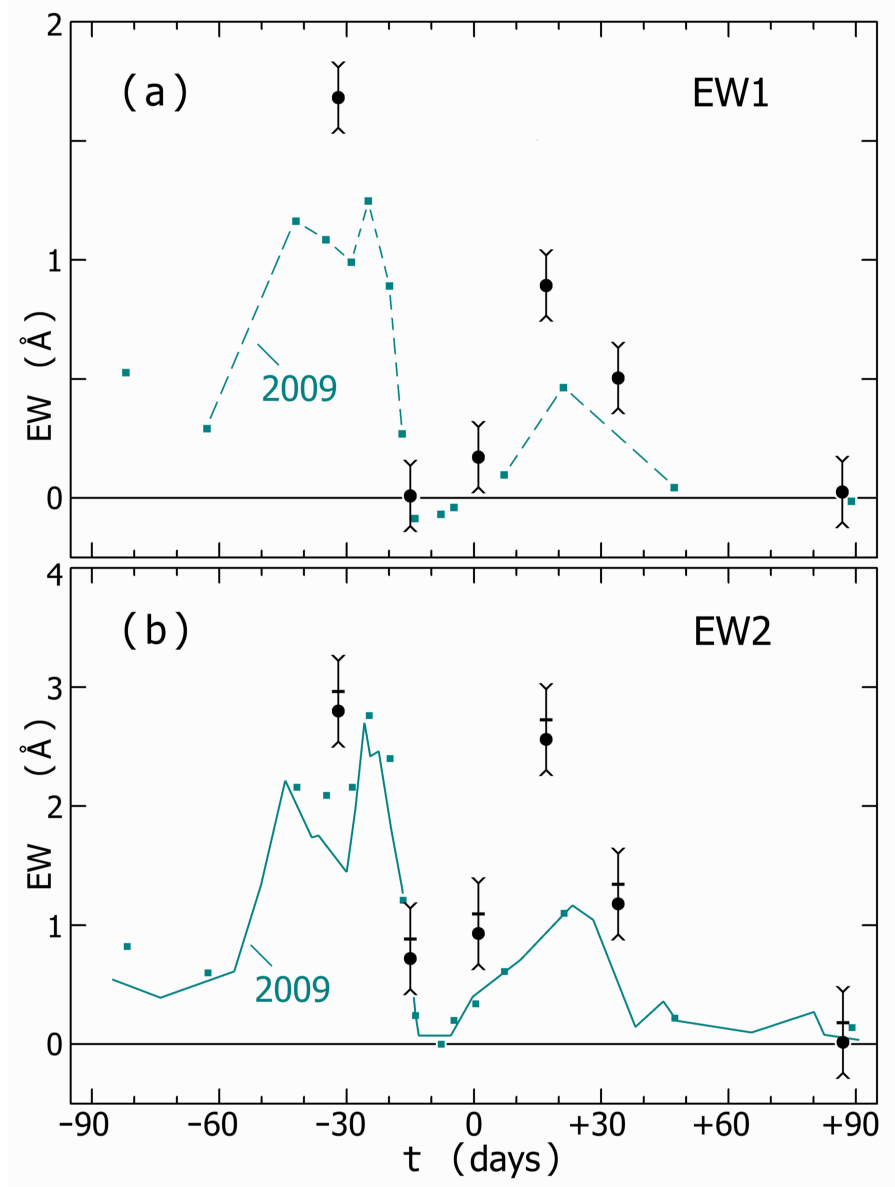

Fig. 3.- EW1 and EW2 for the emission near $4687 \AA$ in 2009 and 2014, see text. The continuous curve in the lower panel traces many data points from 2009 reported by Teodoro et al. (2012). Small squares are other data points for 2009 from Mehner et al. (2011b). The heavy black data points show STIS results in 2014. Their limit-marks represent credible ranges of underlying continua, see text. 
Table 1. STIS Observations of $4561-4841 \AA$ in 2014

\begin{tabular}{cccccc}
\hline \hline Date & $\begin{array}{c}\text { MJD } \\
\text { (days) }\end{array}$ & $\begin{array}{c}\mathrm{t}^{\mathrm{a}} \\
(\text { days })\end{array}$ & $f_{\lambda}(4745 \AA)^{\mathrm{b}}$ & $\begin{array}{c}\mathrm{EW} 1^{\mathrm{c}} \\
(\AA)\end{array}$ & $\begin{array}{c}\mathrm{EW} 2^{\mathrm{c}} \\
(\AA)\end{array}$ \\
\hline $2014-07-13$ & 56851.2 & -31.8 & 1.74 & 1.68 & 2.77 \\
$2014-07-30$ & 56868.1 & -14.9 & 1.99 & 0.01 & 0.69 \\
$2014-08-15$ & 56884.3 & +1.3 & 1.47 & 0.17 & 0.90 \\
$2014-08-31$ & 56900.4 & +17.4 & 1.45 & 0.89 & 2.53 \\
$2014-09-17$ & 56917.0 & +34.0 & 1.53 & 0.51 & 1.15 \\
$2014-11-09$ & 56970.7 & +87.7 & 1.54 & 0.02 & 0.01 \\
\hline
\end{tabular}

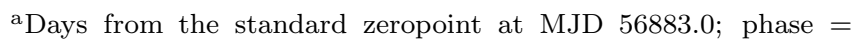
$t /(2023 \mathrm{~d})$.

${ }^{\mathrm{b}}$ Apparent continuum in units of $10^{-11} \mathrm{erg} \mathrm{cm}^{-2} \mathrm{~s}^{-1} \AA^{-1}$, not corrected for slit throughput; see text.

${ }^{\mathrm{c}} \mathrm{EW} 1$ and EW2 are different types of equivalent width for emission near $4687 \AA$, see text. 